\title{
Comparison of Complex Traffic Junction Descriptions in Automotive Standard Formats
}

\author{
Nikol Krausz ${ }^{1 *}$, Vivien Potó1, János Máté Lógó1, Árpád Barsi ${ }^{1}$ \\ ${ }^{1}$ Department of Photogrammetry and Geoinformatics, Faculty of Civil Engineering, Budapest University of Technology and Economics, \\ H-1111 Budapest, Műegyetem rkp. 3, Hungary \\ * Corresponding author, e-mail: krausz.nikol@emk.bme.hu
}

Received: 20 May 2021, Accepted: 19 November 2021, Published online: 03 December 2021

\begin{abstract}
Autonomous and highly automated transportation is very attractive not only for the automotive but also for the mapping industry. In order to exploit the technology, the latest survey solutions are needed, but beyond that, a clear description of the content is a must. Three standards have been selected: (1) used for a long time in navigation systems (NDS), (2) developed for simulation purposes (OpenDRIVE), and (3) designed and proposed for general map data exchange (GDF). In this paper, we present the approach of the three standards, then apply the tools of the standards to a specific sample area, a complex traffic junction, and produce maps in the appropriate formats. The evaluation of the pilot site shows that the difficulty of the exchange standard appears to be a serious obstacle. In the process of applying the navigation standard, the personality of the evaluator (the map maker) is also revealed. In the simulation format, the description of reality is gradually improved by including more and more extra elements.
\end{abstract}

\section{Keywords}

road model, standards, automotive simulations, autonomous driving

\section{Introduction}

Car navigation has always been an activity, which has a base on maps or, later, map databases. For now, the permanent evolution of the navigation maps has reached the state, where the accuracy and resolution can effectively support the onboard vehicular assistants. The trends in this evolution show a high potential also in the expected autonomous driving [1-4].

The increasing levels of automation request better environmental sensing, coupled with enhanced models describing the road infrastructure, the built and natural objects, and also the traveling vehicles and the walking pedestrians. The increased use of computer technologies resulted in rapid development nowadays. Simulation is maybe the best workhorse of this procedure [5-7]. The environmental database - which is called a map - has experienced a sharp paradigm change. Now it must fulfill the strict requirements, which belong to the usual regulations in the automotive industry. The requirements generally have the standards as a realization form.

\section{Automotive standard formats}

The automotive industry has seen a significant amount of growth over the past decade. Challenges stemming from economic and environmental factors have changed the way vehicles must perform, and automotive manufacturers have been required to adapt to them. The challenges are as follows:

- enhanced safety requirements,

- growing demand for automated driving,

- eco-driving, etc.

A self-driving car relying on sensors alone would not be able to understand and follow the rules of the road. The increasing levels of automation request better environmental and road infrastructure information. A map for automated driving needs to allow the precise positioning of a vehicle on the road. A map that meets these requirements is a high-definition map or briefly HD map.

There are two main groups for map data formats:

- physical data formats, which are primarily to store and use the information in the maps, and

- exchange data formats, which enables a gateway between the physical formats.

The physical map data formats can store the information content in binary or (ASCII) text form. The latter is XML (Extensible Markup Language) or JSON (JavaScript Object Notation) in most cases. 
The most frequently used exchange data format is the Geographic Data File (GDF) [8-10], which has actually version 5.1, and was released in 2020 as ISO 20524 standard. The scope of GDF has been declared as "a system for the interchange of digital road-related geographic information". To reach this goal, "all the requirements of applications in the road transport and traffic telematics (RTTT) field" are considered [11].

\section{Navigation Data Standard}

The Navigation Data Standard (NDS) is a standardized format for automotive-grade navigation databases jointly developed by automobile manufacturers, system and map suppliers, but it is also an association registered in Germany [12]. NDS is a standardized physical storage format for navigation systems; the binary database format allows furthermore even the exchange of data between different systems. System suppliers often provide physically or logically separate data collections; NDS defines a database as the totality of navigational data. The content and structure of this database are standardized. NDS separates software from data, thus enhancing flexibility for creating various navigation products for the end-users.

The NDS association has defined several clusters of map data. These are called NDS Building Blocks, supporting the different use cases. Specific building blocks are identified, data are layered and referencing each other (Fig. 1).
DataScript is a language for modeling binary datatypes, bitstreams, or file formats. Using such formal language for defining binary datatypes resolves all ambiguities typically found in textual or tabular specifications $[13,14]$. In addition, one can automatically generate encoders and decoders for a given binary format from this formal specification. NDS has enhanced the DataScript language with relational extensions for describing binary data structures, called Relational DataScript (RDS). RDS enables the definition of hybrid data models: high-level structures are modeled as relational tables and indices in RDS, and the low-level bulk data is stored in Binary Large Objects (BLOBs) in single columns with a format defined in DataScript [15].

The following data types are distinguished in the database:

- Features: All real-world objects relevant to a navigation system are represented by one or more features, on one or more levels.

- Attributes: Attributes describe the specifics of the different features.

- Metadata: Metadata contains information on various database content and database properties. It can refer to a specific product database, a building block, or the complete database.

NDS defines attributes either as fixed or flexible. Fixed attributes are mandatory information to define a feature and to store a value coded compactly, as the attribute-value structure is predefined within the feature class. An example

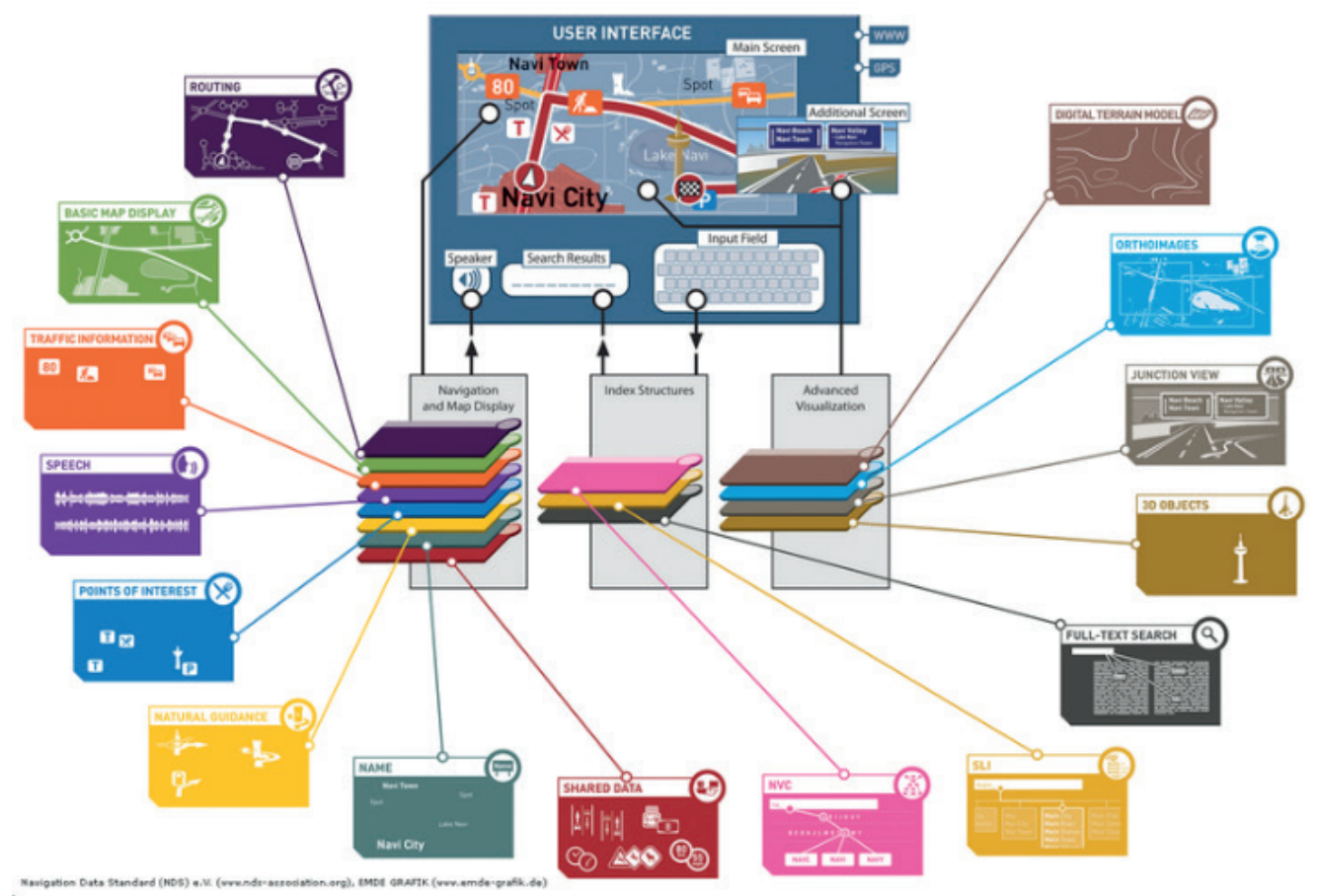

Fig. 1 NDS clusters and data layers 
of a fixed attribute is the link type. Flexible attributes are additional or exceptional information about a feature. They are stored as maps with a tile and may be assigned to one or multiple features in the tile. NDS uses a global tiling scheme, forming a uniform grid that divides the whole surface of the Earth into tiles, thus partitioning the available data.

A link describes a road segment between two intersections. It represents a road or a carriageway. Each link has a start point and an endpoint, but its direction does not describe the traffic flow direction. NDS differentiates two types of links:

- Base links: links located on one tile only,

- Route links: links that extend over more than one tile.

Geometry and topology information of base links is stored directly with the link. A base link feature has its complete geometry, and its intersections are located within one tile. Links that are situated on more than one tile are called route links.

Lanes are numbered separately for each driving direction from the curbside to the middle of the road. Right-hand traffic lanes are numbered from right to left concerning the driving direction, whereas left-hand traffic lanes are numbered vice versa. The numbering starts from 0 (Fig. 2).

A lane group is a set of one or more lanes. All lanes in lane group have the same travel direction, and are ordered from the curbside to the middle of the road. Lane widths in one lane group may vary. The lane connectivity describes the possible maneuvers. Lanes in two lane groups are connected by a connector having the same ID of both lane groups and ensuring non-overlapping lane geometry. An application can derive the connectivity of lane groups in a longitudinal direction from the validity ranges assigned to the base link or road geometry line. To ensure lane connectivity at intersections, the database shall assign all base links that start or end at an intersection to the intersection. The intersection association indicates that traffic from the intersecting links can affect traffic on all connected lanes. Fig. 3 illustrates possible transitions within an intersection. This figure simplifies the lane groups that represent the parts of the base links that bring traffic into the intersection.

\section{OpenDRIVE}

OpenDRIVE is a member of the Open Standards family, initially created by a German company named VIRES Simulationstechnologie GmbH. OpenDRIVE was the first open format to prescribe the logical descriptions of roads and road networks in 3D environments [16]. The OpenDRIVE standard has been reviewed and released by a core team of

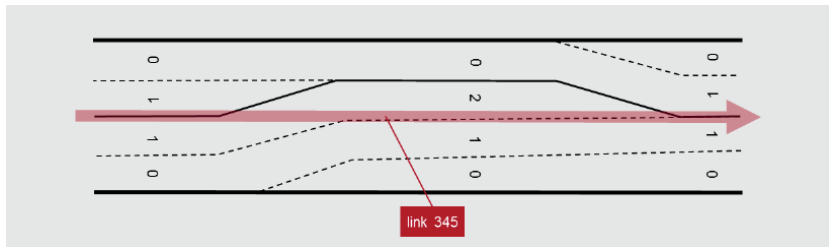

Fig. 2 Numbering lanes in NDS

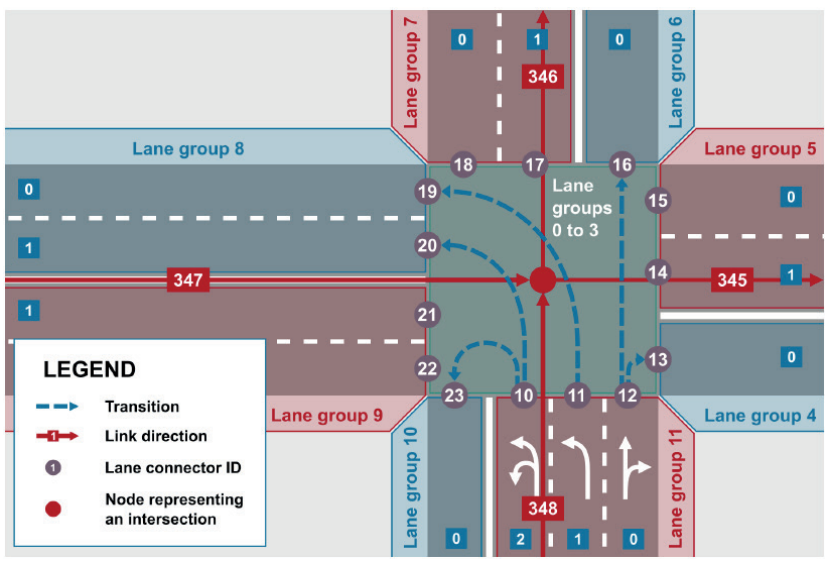

Fig. 3 Connectivity of lanes across an intersection

driving simulation experts since 2006. This team decided to take OpenDRIVE to the next level of professionality by transferring management to ASAM e.V. (www.asam.net) on September 7th, 2018. The latest released version is 1.7.

The inventors have realized that roads are similar throughout all systems and countries, and the elements can't be proprietary. Therefore, potentially suitable support to driving simulators must consider standardized format (for the data storage and the information visualization) and - at the same time - has to own a proprietary format to express the logical model.

A requirement system has been formulated to achieve these goals:

- the new data format must be able to be international,

- country-specific elements should be avoided, or a certain generalization is needed,

- the description technique must be state-of-the-art,

- the focus must be on driving simulator applications,

- the data format must have extensibility,

- users must be able to customize the model without any interference with elements,

- it must be publicly available,

- the format must be without any licensing,

- users must be involved in developing future releases, and

- all suggestions or new requirements must have a defined process. 


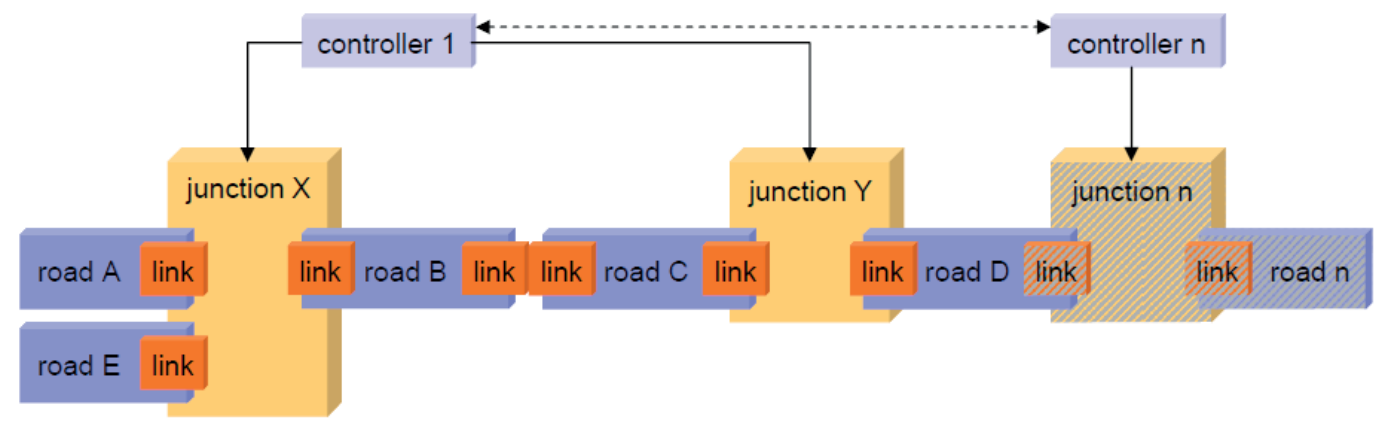

Fig. 4 Principle of road connection in OpenDRIVE

The crucial elements of the standard are roads, junctions, and controllers. Roads and junctions are glued together with links. Fig. 4 demonstrates this principle:

The listed requirement of extendibility, readability, and state-of-the-art description is fulfilled by XML technology: the standard prescribes the XML format since version 0.7 (originally it was a binary format). The adequate extension is .xodr for the uncompressed and .xodrz for the compressed version in rare cases.

The road is the central part of the model description. This section stores all road elements. There is a reference line definition, which is composed of lines, arcs, clothoids (here: spirals), or parametric curves (here: cubic polynomials) (Fig. 5(a)). These components ensure the smooth and exact definition of the road's horizontal design. Beyond the horizontal definition, the road is also specified vertically (by longitudinal and lateral profiles, superelevations, etc.). Along the reference line, various properties of the road can be defined. It must be emphasized that the topology has primer importance: the roads in a model must be connected properly, stored by the road links. Roads are composed of lanes that can be split into homogeneous lane sections. Generally, lanes are unique elements described in sequence, starting from the reference line and ascending to the left direction (positive lane IDs), descending to the right (with negative IDs). These rules are applied in all lane sections (Fig. 5(b)). Lanes can be shifted by defining offset values, or lane numbers can be varied in a lane section (adding more lanes or deleting lanes).

The junction is an area where three or more roads meet. They are crucial elements in road network description, especially because the correct topology is significantly more complicated than regular road segments. As a complex example, Fig. 6(a) demonstrates that the incoming roads can be one-way or two-way roads; they can have one or more lanes, and naturally, the figure of the junction can have an arbitrary form. The junction must ensure the correct connection among roads, but also lanes. The

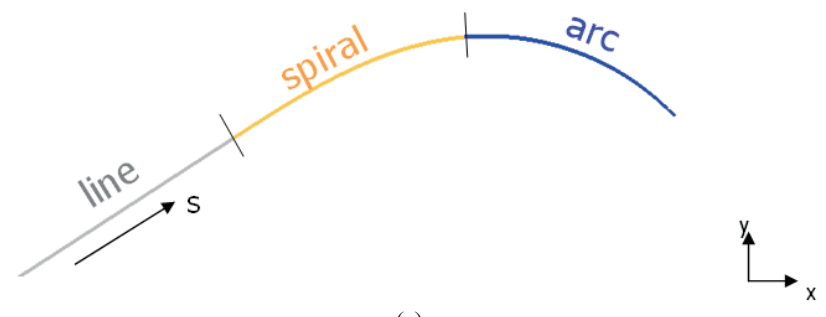

(a)

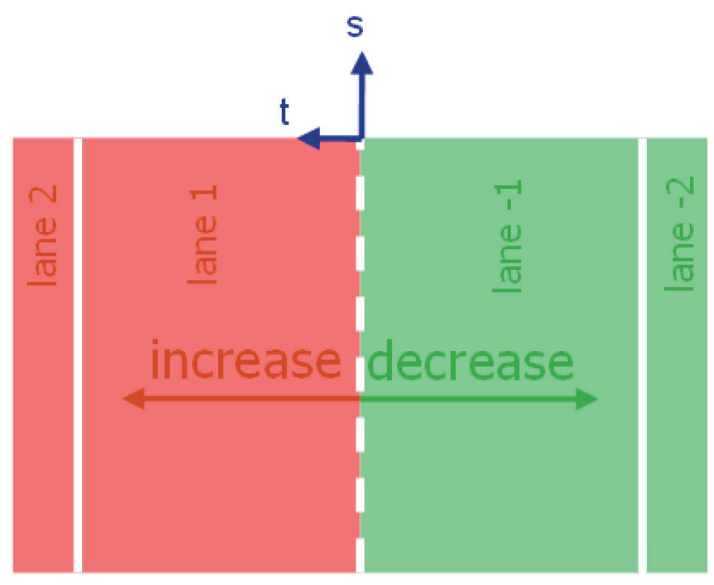

(b)

Fig. 5 a) The road reference line, b) The lane numbering rule

challenge is that these connections are not only covered by simple "graph edges", but the usual road elements must define these connections geometrically. Roads of a junction have similarly reference line forming special pattern (Fig. 6(a)). Fig. 6(b) demonstrates the variety of connectivity of one incoming direction within a junction.

One can imagine that a more complex junction has expressively more overlapping connections. The junction topology is stored practically as the lane level links in predecessor and successor tags (similarly to road level links). These tags are crucial in the geometry preparation for any simulation procedure. A junction group is a composite element in OpenDRIVE, where a compound road connection can be designed and handled. Typically, roundabouts and largely extended complex junctions are described in this manner. 


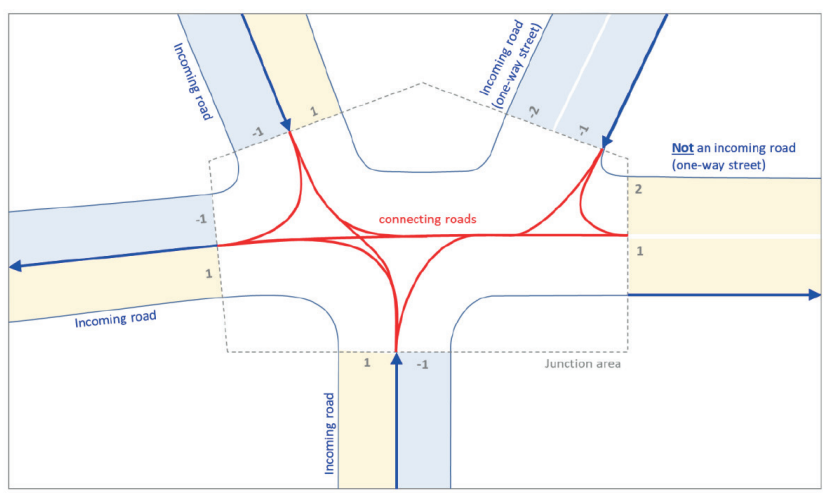

(a)

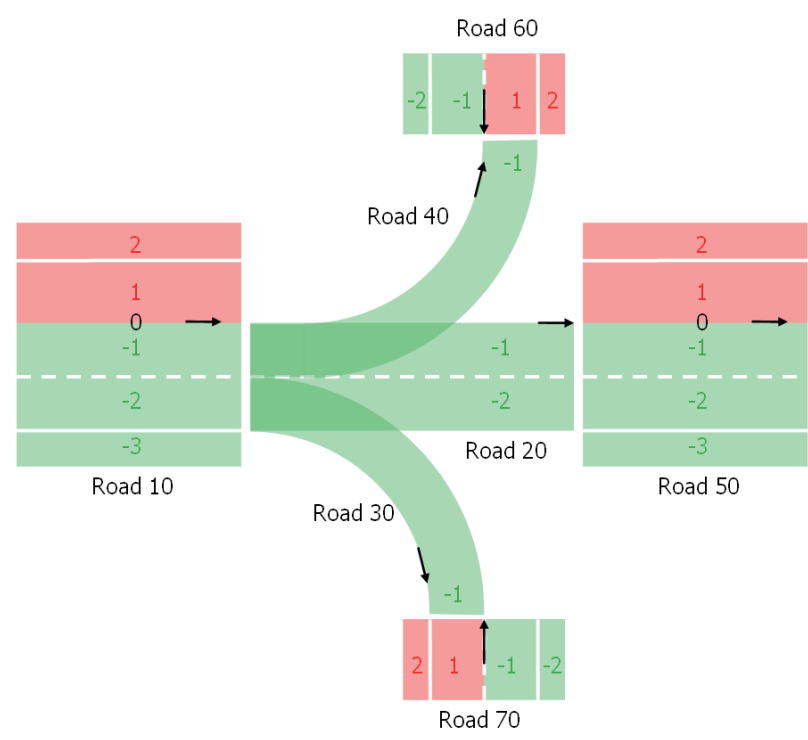

(b)

Fig. 6 a) A complex road junction with five incoming roads, b) The OpenDRIVE junction schema with lanes

\section{Geographic Data File}

The Geographic Data File (GDF) is a standardized, application-independent exchange file format for geographic data used to define and interchange digital road databases for navigation applications designed by the ISO/TC204 (Intelligent Transport System) [9, 10]. GDF provides detailed rules for data capture and representation, and an extensive catalog of features, attributes, and relationships.
The different objects together making up a GDF are conceptually divided over three different levels: Level-0, Level-1, and Level-2. The topological primitive building blocks - node, edge, and face - reside on Level-0. The simple features - point, line, and area - and the data models make up Level-1. Simple features may be aggregated into complex ones comprising Level-2. GDF supports three different types of graph topologies: planar, non-planar, and non-explicit topologies:

- Non-explicit topology: No topological relations between objects are explicitly defined, where these relations are only defined via coordinate values.

- Non-planar topology: Topological relations are explicitly defined; objects may entirely or partially coincide, intersect or overlap.

- Planar topology: Topological relations are explicitly defined; objects shall not fully or partially coincide, shall not intersect or overlap. Intersecting objects or overlapping objects are broken up into object fragments, whereby common parts (such as a street coinciding with a boundary) are present only once.

The features are the central element in the data model, where they are database representations of real-world geographic objects. Conceptually GDF defines a basic data model to describe the road and road-related objects. Roads and intersections are expressed by lines and points, while the level is used to unify bidirectional road elements to a single road.

The core is a belt concept, which is a specific area feature (Fig. 7). A belt is composed of two sidelines and is terminated by two terminal lines. Belts can be used both on the road and on the lane modeling levels, too.

RoadBelt represents the area of the road that includes carriageways, median (island) and sidewalks (Fig. 8), where RoadBeltElement characterizes a section of the road used for the movement of vehicles, placed between a pair of IntersectionBelt Feature. A road between intersections is expressed by a single RoadBeltElement or dual

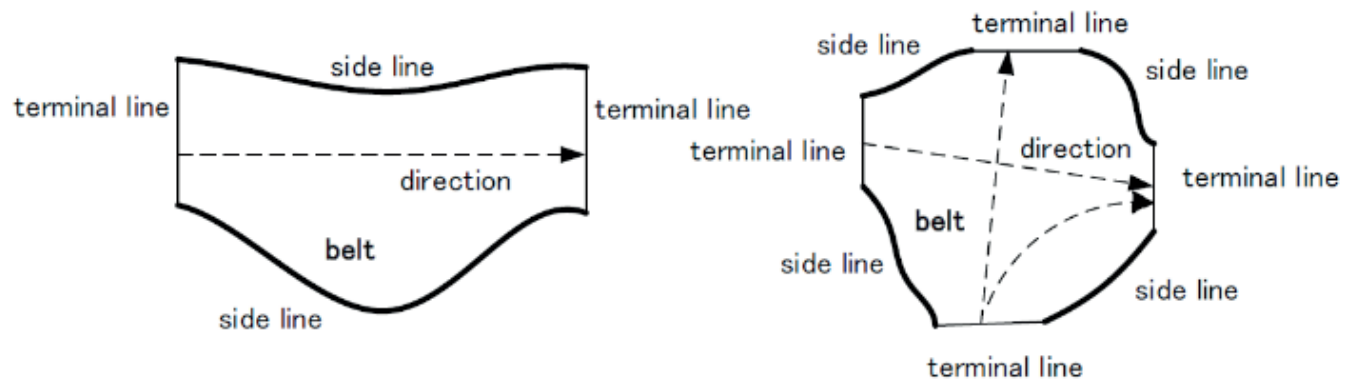

(a)

(b)

Fig. 7 GDF belt concept to express the basic data model a) RoadBelt, b) IntersectionBelt 


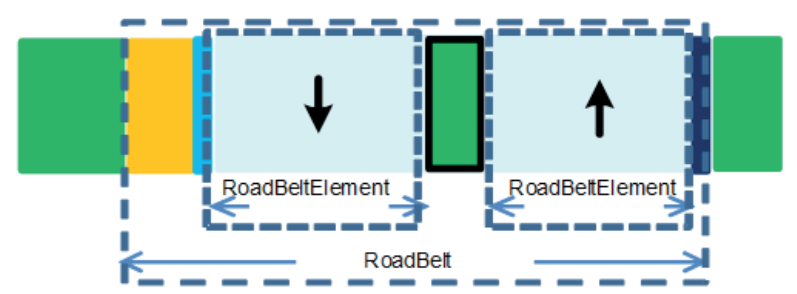

Fig. 8 Concept for dual carriageway with sidewalk with GDF Roadbelt and RoadBeltElement

RoadBeltElement. RoadBeltElements shall not overlap an IntersectionBelt. Sidelines of a RoadBeltElement are defined by outer lines of the carriageway, road markings along the road indicating the edge of the carriageway at the median, and/or boundary lines for traffic controls. Terminal lines of a RoadBeltElement are virtual lines and are defined at the same location of the terminal lines of an IntersectionBelt. RoadBeltElement serves as the smallest unit for representing an independent road element.

\section{Descriptions of a sample junction}

The above presented standards are hard to understand without any practical examples, therefore we have selected a pilot site, which is in the vicinity of our research group and is enough representative to demonstrate the strengths and weaknesses, or the advantages and problems during the map database creation. The Szent Gellért square (Fig. 9) is located in the district Budapest XI between Gellért Hill and the Danube. The square is a busy place with several connecting roads, streets, quays, where not only public roads but also tram lines, a tram and bus stops, as well as a bridge entry can be found. This area has all the complexity, which can efficiently prove the usage of the above-written standards.

An orthoimage enables the delineation of the paved and green surfaces, as well as it helps in differentiating between the lanes, sidewalks, and all elements, which are regulated in the standards. Further positive feature of an orthophoto is its true (metric) scale and precisely georeferenced representation, so the derived models are ready for direct simulation inputs.

\section{Comparison of the standards}

The main goal of the current analysis was to examine the similarities and differences of the map representations prepared for the sample junction area considered the presented standards. Knowledge of the place, the local traffic rules helped us a lot in our work.

All three described standards have similarities in constructing a complex road model from basic building blocks. The main parameters are summarized in Table 1.

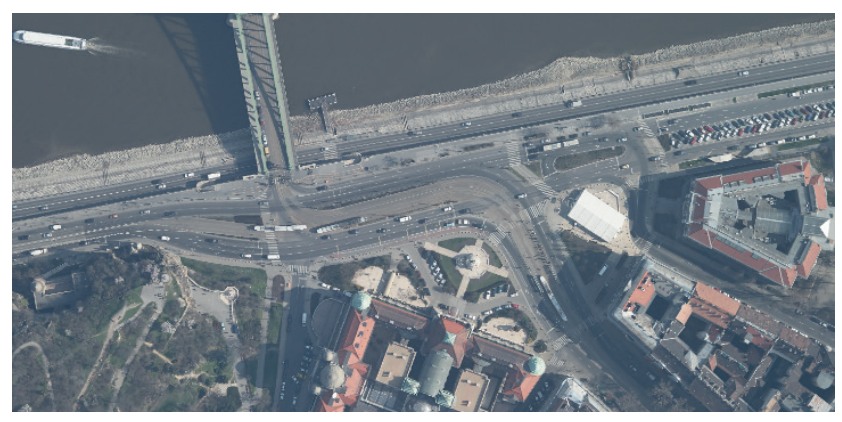

Fig. 9 The orthoimage of the Szent Gellért square in Budapest

Table 1 Comparison of the standards

\begin{tabular}{|c|c|c|c|}
\hline & OpenDRIVE & NDS & GDF \\
\hline Version & 1.4 & 1.0 & 5.1 \\
\hline $\begin{array}{l}\text { Designed } \\
\text { purpose }\end{array}$ & $\begin{array}{l}\text { Driving } \\
\text { simulator }\end{array}$ & $\begin{array}{c}\text { Embedded } \\
\text { car navigation } \\
\text { devices }\end{array}$ & $\begin{array}{c}\text { Exchanging } \\
\text { between various } \\
\text { data storage } \\
\text { formats }\end{array}$ \\
\hline $\begin{array}{l}\text { Road } \\
\text { geometry }\end{array}$ & $\begin{array}{c}\text { A reference line, } \\
\text { as lines, spirals, } \\
\text { arcs, and cubic } \\
\text { polynomials }\end{array}$ & $\begin{array}{l}\text { Single polyline } \\
\text { with start and } \\
\text { end nodes }\end{array}$ & $\begin{array}{c}\text { A complex } \\
\text { feature } \\
\text { consisting of } \\
\text { simple features } \\
\text { or other complex } \\
\text { features }\end{array}$ \\
\hline $\begin{array}{l}\text { Lane } \\
\text { geometry }\end{array}$ & $\begin{array}{l}\text { Offset from the } \\
\text { reference line, } \\
\text { variable lane } \\
\text { width }\end{array}$ & $\begin{array}{l}\text { Centerline and } \\
\text { lane boundaries, } \\
\text { by 3D vectors, } \\
\text { NURB splines }\end{array}$ & $\begin{array}{l}\text { Constant lane } \\
\text { width and curve } \\
\text { by line segments }\end{array}$ \\
\hline $\begin{array}{l}\text { Coordinate } \\
\text { system }\end{array}$ & $\begin{array}{c}\text { WGS } 84-\text { EPSG } \\
4326\end{array}$ & WSG 84 & WSG 84 \\
\hline Structure & $\begin{array}{l}\text { Hierarchical } \\
\text { structure }\end{array}$ & Multilayers & $\begin{array}{c}\text { Datasets, layers, } \\
\text { and sections }\end{array}$ \\
\hline Format & XML & $\begin{array}{l}\text { SQLite and } \\
\text { Relational } \\
\text { DataScript }\end{array}$ & XML \\
\hline
\end{tabular}

The fundamental similarity in the standards is their reference line usage in relation to building blocks. The road geometry uses an ordered sequence of shape points describing the geometry of a polyline (or a simple line) that represents the course of a road, where each road section has its start and end nodes represented as a simple graph [17]. An intersection is interpreted as a vertex of the graph, whereas a road is an edge. Reference lines may be produced both at road and lane levels independently. Descriptive attributes are assigned to building blocks and can be selected from a specific list. Navigation systems for highly automated driving need detailed information on road topology and road geometry; therefore, lanes are necessary for traffic applications (lane-change and merging). The main road elements are the centerline/link and the lane description. Differences can be found in the object relationships and in the way they are stored. 
In NDS, the first road representative line is a link, which is a simple graph. Route links are purely topological routing features. Approaching the more detailed road information, the standard also assigns a centerline to the roads and lanes. It also specifies the connection of the lanes and groups of lanes with lane connector points and a lane numbering method. This way, it is possible to avoid overlaps and gaps in the digital representation of the road.

We carried out a test: all authors evaluated the intersection based on own NDS interpretation. There were differences in the connection interpretations according to the standard and also alterations in the dividing lines placement to delimit the intersections, whether or not it is allowed to turn from one lane to each connecting lane in the case of a multi-lane road. Similar to this, alterations were found in handling bus stops, which are just a piece of road without any road mark. The evaluations were stacked for comparison; based on consolidated rules the final map has been completed. Map gaps were resolved using the assistance of the multinational map provider company NNG. Eventually, due to its complexity, tram line was removed from the mapping process (Fig. 10).

Because GDF has no specific viewer application; it has been designed to interface the different physical formats, we didn't prepared any illustration to the GDF. As it was stated, GDF provides a base for capturing and exchanging geographic content and has similarities to the NDS structure. It has the BeltRepresentativeLine for roads and LaneRepresentativeLine for lane centerlines.

In contrast, the OpenDRIVE standard uses centerlines (reference line) as a base, and all the elements describing the road are attached to. The pilot site was evaluated by Mathworks RoadRunner software. The tool was capable to export OpenDRIVE compatible format. The

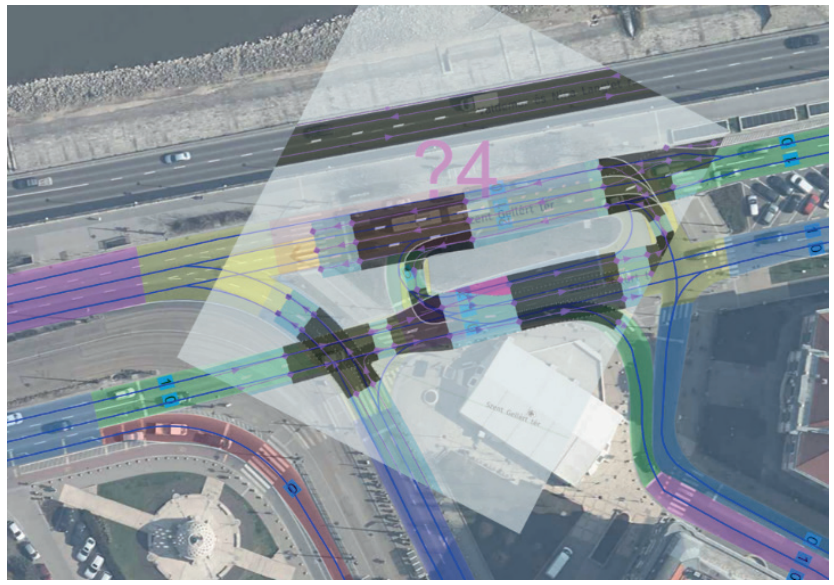

Fig. 10 NDS representation spots in the pilot site simplest visualization screen during the digitization process is demonstrating the OpenDRIVE's lane philosophy (Fig. 11). The software has a built-in graphical engine not only to show photorealistic views but also has the export option in diverse application formats (Fig. 12).

Some numerical comparisons can underline the above-illustrated differences: the NDS description of the test area is composed of 9 intersections and 33 lane groups. At the same time, GDF applies 11 IntersectionBelts and 23 RoadBelts to achieve the exact same details. It is evident that this example is not representative but demonstrates the alterations in the standardized formats.

\section{Conclusions}

Simulation has a primary role in the vehicular development process as it enables cost- and time-efficient testing. In autonomous driving, comprehensive knowledge about road network data is a necessity. These simulation applications require accurate road geometric and attribute description that needs to be created and shared in a common format.

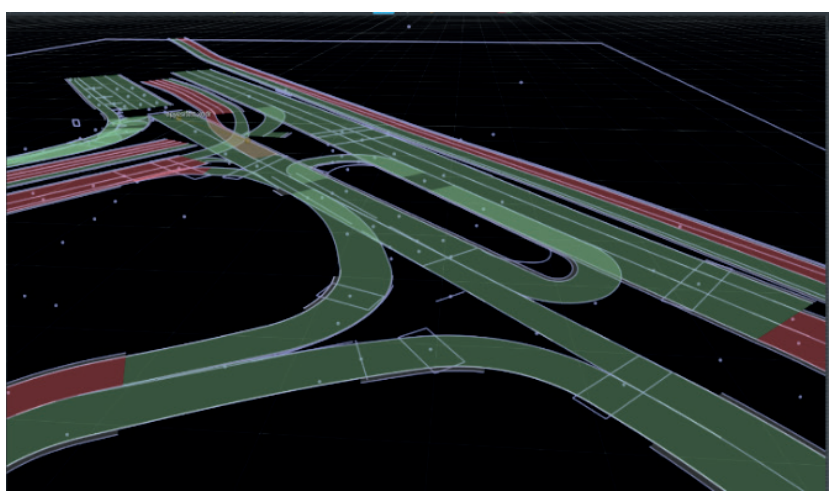

Fig. 11 OpenDRIVE lanes in RoadRunner for the pilot site

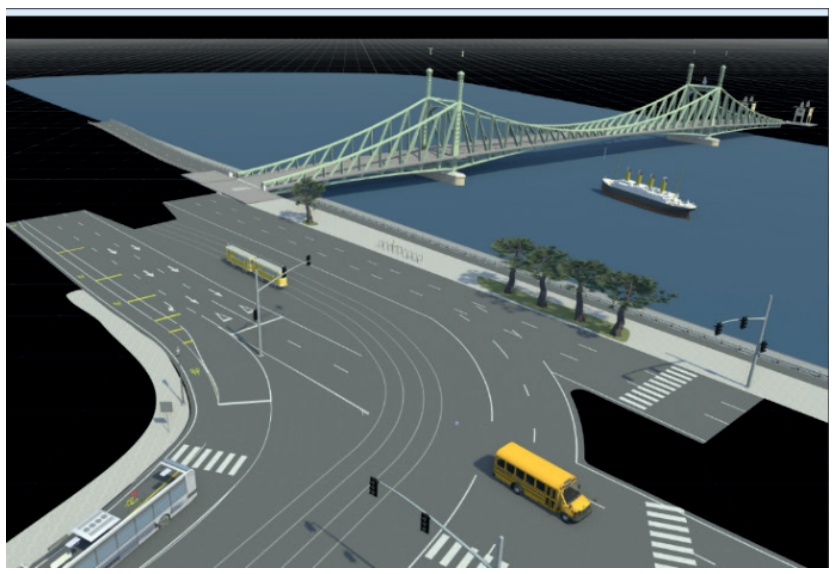

Fig. 12 RoadRunner photorealistic visualization with built-in and importable Sketchup models 
The documentation of the GDF standards is almost 2,000 pages, but with merely a few figures of the general solution for intersections that can be mapped in multiple ways. The presented standards contain a detailed description of the definition of the road and related ancillary elements, furthermore recommendation about their database storage methodology. Only a small number of examples are given for simple intersection types, lane separations, merging.

The shown NDS standard doesn't limit the personal interpretations and rule applications, primarily visible in the turning options. The digitizer has quite a lot of freedom in deciding how the standard is realized in concrete situations; his/her personal driving experiences greatly influences the modelling thinking. Sometimes the general rule of thumbs or simply traditions are followed in developing the lane level topology. Three- (T) or four-arm (X) junctions can be mentioned as examples. This format is frequently used by several map providers unlike GDF, which remained a theoretical conversion option. It is expected that the next GDF version will have significantly clearer approach, maybe supporting software or code to ease its spread.

\section{References}

[1] Szalay, Z., Nyerges, Á., Hamar, Z., Hesz, M. "Technical Specification Methodology for an Automotive Proving Ground Dedicated to Connected and Automated Vehicles", Periodica Polytechnica Transportation Engineering, 45(3), pp. 168-174, 2017. https://doi.org/10.3311/PPtr.10708

[2] SIP "SIP-adus Innovation of Automated Driving for Universal Service", [online] Available at: https://en.sip-adus.go.jp/

[3] Németh, H., Háry, A., Szalay, Z., Tihanyi, V., Tóth, B. "Proving Ground Test Scenarios in Mixed Virtual and Real Environment for Highly Automated Driving", In: Mobilität in Zeiten der Veränderung, Springer Gabler, Wiesbaden, Germany, 2019, pp. 199-210. https://doi.org/10.1007/978-3-658-26107-8 15

[4] Szalay, Z., Tettamanti, T., Esztergár-Kiss, D., Varga, I., Bartolini, C. "Development of a test track for driverless cars: Vehicle design, track configuration, and liability considerations", Periodica Polytechnica Transportation Engineering, 46(1), pp. 29-35, 2018. https://doi.org/10.3311/PPtr.10753

[5] SAE "J3016 Warrendale, PA, USA, 2018.

[6] Burg, M. "Simulation as Tool Continuous Software Testing with Simulation while Development Phase" [pdf] Continental Teves AG \& Co. oHG, Frankfurt am Main, Germany, 2018. Available at: https:// www.ko-haf.de/fileadmin/user_upload/media/abschlusspraesentation/18_Ko-HAF_Simulation-as-Test-Tool.pdf

[7] Kang, Y., Yin, H., Berger, C. "Test your self-driving algorithm: An overview of publicly available driving datasets and virtual testing environments", IEEE Transactions on Intelligent Vehicles, 4(2), pp. 171-185, 2019.

https://doi.org/10.1109/TIV.2018.2886678
OpenDRIVE is a standard of highly increasing popularity. More and more simulation and visualization software accept its format as input; simultaneously more sophisticated modelling environments, like RoadRunner (originally from VectorZero, now from Mathworks after its acquisition) offering the output map format considering OpenDRIVE standard. The open nature of the standard guarantees the legal purity of its use, which might increase furthermore the attractiveness of the format.

\section{Acknowledgement}

The research reported in this paper and carried out at the Budapest University of Technology and Economics has been supported by the National Research Development and Innovation Fund (Grant TKP2020 Institution Excellence Subprogram, No. BME-IE-MIFM and Grant ÚNKP21-3-II-BME-26) based on the charter of bolster issued by the National Research Development and Innovation Office under the auspices of the Ministry for Innovation and Technology. The project has been supported by the European Union, co-financed by the European Social Fund. EFOP-3.6.3-VEKOP-16-2017-00001.

[8] ISO "ISO 14825:2011 - Intelligent transport systems - Geographic Data Files (GDF) - GDF5.0", International Organization for Standardization, Geneva, Switzerland, 2011. [online] Available at: https:// www.iso.org/standard/54610.html [Accessed: 31 January 2020]

[9] ISO "ISO 20524-1:2020 - Intelligent transport systems Geographic Data Files (GDF) GDF5.1 - Part 1: Application independent map data shared between multiple sources", International Organization for Standardization, Geneva, Switzerland, 2016. [online] Available at: https:/www.iso.org/standard/68244.html [Accessed: 17 May 2020]

[10] ISO "ISO 20524-2:2020 Intelligent transport systems - Geographic Data Files (GDF) GDF5.1 - Part 2: Map data used in automated driving systems, Cooperative ITS, and multi-modal transport", International Organization for Standardization, Geneva, Switzerland, 2020. [online] Available at: https://www.iso.org/standard/72494.html [Accessed: 17 May 2020]

[11] ISO "ISO/TR 14825:1996 Geographic Data Files (GDF)", International Organization for Standardization, Geneva, Switzerland, 1996. [online] Available at: https://www.iso.org/standard/25627. html [Accessed: 18 May 2020]

[12] Navigation Data Standard "The worldwide standard for map data in automotive eco-systems", [online] Available at: https://www. nds-association.org/ [Accessed: 29 October 2018]

[13] Back, G. "DataScript-a Specification and Scripting Language for Binary Data", In: Proceedings of the ACM Conference on Generative Programming and Component Engineering Proceedings (GPCE 2002), Pittsburgh, PA, USA, 2002, pp. 66-77.

https://doi.org/10.1007/3-540-45821-2_4 
[14] Wellmann, H. "DataScript Language Overview", Harman/Becker Automotive Systems, Stamford, CT, USA, 2006. [online] Available at: http://dstools.sourceforge.net/DataScriptLanguageOverview.pdf

[15] Navigation Data Standard "NDS Open Lane Model webpage", [online] Available at: http://www.openlanemodel.org/ [Accessed: 31 January 2019]
[16] ASAM "ASAM OpenDRIVE Transfer Project", [online] Available at: https://www.asam.net/project-detail/asam-opendrive-transfer-project/ [Accessed: 06 April 2018]

[17] Bétaille, D., Toledo-Moreo, R. "Creating Enhanced Maps for Lane-Level Vehicle Navigation", IEEE Transactions on Intelligent Transportation Systems, 11(4), pp. 786-798, 2010. https://doi.org/10.1109/TITS.2010.2050689 